\title{
Diagnóstico, Definição e Melhoria do Processo de Software: um Estudo de Caso
}

\author{
Débora P. Diniz Tavares \\ Sandra C. P. Ferraz Fabbri \\ Universidade Federal de São Carlos \\ Departamento de Computação \\ Rodovia Washington Luis, $\mathrm{km} 235$ \\ Cx. Postal 676, 13565-905, \\ São Carlos, SP, Brasil \\ \{debora,sfabbri\}@dc.ufscar.br
}

\author{
Rosely Sanches \\ Universidade de São Paulo \\ Instituto de Ciências Matemáticas e Computação \\ Av. do Trabalhador São-Carlense, 400, \\ Centro,Cx. Postal 668, 13560-970, \\ São Carlos, SP, Brasil \\ rsanches@icmc.usp.br
}

\begin{abstract}
Resumo
Este artigo relata um estudo de caso realizado em uma empresa de internet, telecomunicações e desenvolvimento de Website, que teve por objetivo avaliar e identificar o processo atual de desenvolvimento de software da empresa e propor um plano de melhoria desse processo, com base no nível 2 do modelo SW-CMM. Para atingir esse objetivo, foi utilizado um ferramental gerado por três trabalhos de mestrado desenvolvidos nos Grupos de Engenharia de Software da UFSCar e do ICMC-USP. Esses trabalhos correspondem a um questionário de avaliação de processo segundo o nível 2 do SW-CMM, uma ferramenta que dá apoio automatizado a esse questionário e um conjunto de diretrizes que ajudam a estabelecer um plano de ação para iniciar a melhoria de processo.

Palavras-Chave: Diagnóstico de Processo, Melhoria de Processo, SW-CMM.
\end{abstract}

\section{Abstract}

This paper describes a case study realized in an company whose business is related to internet, telecommunications and Website development. The objective of this case study was to evaluate and to identify the actual software development process of this company and to propose a plan to improve this process, based on level 2 of the SW-CMM model. To reach this objective it was used instruments generated by three Ms. works developed by the software engineering groups from UFSCar and ICMC-USP. These works correspond to a process evaluation questionnaire based on level 2 of SW-CMM, a tool that supports this questionnaire and a set of guidelines that helps to establish an action plan to initiate the process of improvement.

Keywords: Process Evaluation, Process Improvement, SW-CMM.

\section{Introdução}

Atualmente o software é abordado sob dois pontos de vista: Produto e Processo, sendo que, nos dias de hoje, existe uma conscientização de que a qualidade do produto de software é fortemente determinada pela qualidade do processo utilizado durante o seu desenvolvimento e manutenção [1]. Dada a importância do processo na qualidade do produto, vários trabalhos têm abordado esse tema, existindo inclusive ferramentas que apóiam a definição do processo de desenvolvimento de software [2,3].

Em relação à qualidade do processo propriamente dita, têm sido propostos vários modelos que podem ser estabelecidos como ponto de referência, como por exemplo, os modelos SW-CMM [4] e a futura Norma ISO/IEC 15504 [5]. Entretanto, esses modelos, em geral, exigem grandes investimentos financeiros e de infra-estrutura, o que os tornam inviáveis para as empresas de pequeno porte, as quais, no contexto desse artigo e também no contexto de algumas pesquisas [6], são empresas caracterizadas por um número reduzido de funcionários e de recursos. Entende-se, no contexto deste artigo, que tais características 
podem não permitir que esses modelos sejam utilizados por elas na forma como foram propostos. $\mathrm{O}$ estudo de caso apresentado neste artigo teve como objetivo fazer um diagnóstico do processo de desenvolvimento de software de uma empresa de pequeno/médio porte, definir esse processo formalmente e planejar sua melhoria utilizando para isso um ferramental de apoio que pode facilitar iniciativas de melhorias de processo para esse tipo de empresa.

Na Seção 2 apresenta-se uma breve descrição da empresa que participou do estudo de caso. Na Seção 3 descreve-se, resumidamente, o ferramental utilizado neste trabalho. Na Seção 4 descreve-se o estudo de caso realizado e, na Seção 5 apresentam-se as conclusões.

\section{A empresa}

A LinkWay é uma empresa de Prestação de Serviços que atua no segmento de conexão a Internet, desenvolvimento de aplicações para Web e serviços de Telecomunicações, autorizada pela ANATEL (Agência Nacional de Telecomunicações).

A Matriz administrativa da LinkWay é localizada em Rio Claro/SP e suas unidades comerciais estão localizadas em oito cidades do interior de São Paulo. O departamento de pesquisa e desenvolvimento de novas tecnologias fica em São Carlos/SP, no qual a empresa mantém uma equipe de circuito e redes de Telecomunicações e o Centro de Desenvolvimento de Aplicações Web, que são compostos por profissionais qualificados, atualmente responsáveis por Projetos de Conectividade e Soluções de Aplicações na Internet para empresas de pequeno, médio e grande porte de todo o Brasil.

A LinkWay investe continuamente na melhoria de processos de desenvolvimento, buscando com isso um melhor gerenciamento e controle das atividades internas, o que resulta em maior satisfação dos clientes, melhor qualidade dos produtos e aprimoramento profissional dos seus funcionários. A busca pela certificação CMM - nível 2 é a garantia de que a LinkWay é uma empresa empenhada em superar as expectativas de seus clientes satisfazendo assim o crescente mercado da Internet.

\section{Ferramental utilizado no trabalho}

Durante o estudo de caso foram utilizados três trabalhos já concluídos pelos Grupos de Engenharia de Software da UFSCar e do ICMSC-USP com o objetivo de avaliar a utilização conjunta dos mesmos durante um processo de diagnóstico e planejamento de melhoria de processo de desenvolvimento de software. Esses trabalhos serão brevemente comentados a seguir.

\subsection{Questionário de Diagnóstico de Processo de Software com Base no Nível 2 do CMM - QDPS-N2}

Esse questionário foi elaborado com base no modelo SW-CMM para avaliação de processo no que se refere ao nível 2 do modelo, concentrando-se nas sub-práticas das práticas chaves da característica comum Atividades Realizadas necessárias para o atendimento de cada KPA (Key Process Area) que compõe esse nível [7]. Suas perguntas foram elaboradas utilizando-se uma redação menos rígida e mais acessível às pessoas que não estão acostumadas a trabalhar com os termos técnicos do SW-CMM ou mesmo da Engenharia de Software. A contagem é realizada de forma a fornecer um retrato mais fiel do processo da empresa, uma vez que cada sub-prática é computada. Nesse trabalho foi proposta uma tabela de dependência entre as questões para apoiar a identificação de incoerência entre as repostas. 
Além disso, também faz parte do questionário uma lista de questões que tem por objetivo caracterizar o perfil das empresas. O QDPS-N2 foi utilizado no estudo de caso como instrumento de avaliação do processo de desenvolvimento de software da empresa em questão.

\subsection{Ferramenta Software Process Quality - SproQ}

Essa ferramenta auxilia a avaliação de processo de software coletando dados automaticamente de um questionário baseado nas KPA's do nível 2 e em duas KPA's do nível 3 do modelo SW-CMM. A partir desses dados, a ferramenta permite e elaboração de gráficos e relatórios que caracterizam o processo atual da empresa [8]. Como a ferramenta é aberta, isto é, permite instanciar o questionário utilizado, no contexto deste trabalho essa ferramenta foi utilizada apoiando a aplicação do questionário QDPS-N2, uma vez que este foi o instrumento de avaliação para fazer o diagnóstico da empresa durante o estudo de caso.

\subsection{Diretrizes para o Estabelecimento de Melhoria de Processo adequadas a Empresas de Pequeno Porte}

Essas diretrizes têm por objetivos estabelecer prioridades, desenvolver uma abordagem de melhoria e planejar ações de melhoria de Processo de Software para empresas de pequeno porte que buscam alcançar o Nível 2 do SW-CMM [9]. Essas diretrizes seguem uma seqüência de três etapas. Na primeira etapa, é gerado o Relatório de Atividades com as tarefas a serem executadas para o cumprimento de cada KPA que não está sendo cumprida pela empresa. Isso é feito com base nos resultados do diagnóstico do processo atual de desenvolvimento de software, que foi realizado utilizando-se o questionário QDPS-N2. Na segunda etapa, que opcionalmente pode ser realizada em paralelo à fase de diagnóstico, é aplicado o Questionário de Pré-Condições. As perguntas desse questionário foram elaboradas com o objetivo de abordar as condições necessárias, ou pré-condições, para a implementação eficaz do processo de software, as quais envolvem, tipicamente, recursos, estrutura organizacional e treinamento. Com a análise dos dados desse questionário, são gerados dois relatórios: o Relatório de Pré-Condições, que contém as pré-condições necessárias para que a empresa consiga realizar as KPA's e o Relatório de Prioridades, que contém informações sobre quais KPA's possuem maiores condições de serem realizadas pela empresa, visto que as pré-condições que a empresa já possui estão mais próximas daquilo que ela precisaria para realizar essas KPA's. Essas informações auxiliam a gerência no estabelecimento de prioridades das tarefas de melhoria. Na terceira etapa, é elaborado o Plano de Ação, para cada KPA, com base nas informações dos relatórios de Atividades e de Pré-Condições.

\section{Descrição do Estudo de Caso}

O estudo de caso realizado na LinkWay teve como objetivo dar início à melhoria de seu processo de desenvolvimento de software com base no nível 2 do modelo SW-CMM, conforme era desejo da empresa. Nesse sentido, duas atividades foram executadas inicialmente: o diagnóstico da situação atual e a definição do processo da empresa. Após essas atividades, foi definido um plano de melhoria para a empresa.

\subsection{Diagnóstico da situação atual e definição do processo da empresa}

Para se conseguir melhorar um processo de software é necessário conhecer a situação 
em que ele se encontra, o que é possível após realizar um diagnóstico do mesmo. Da mesma forma, é necessário ter esse processo definido, para que todos na empresa possam trabalhar de forma homogênea.

\section{- Diagnóstico da situação atual da empresa}

Para realizar o diagnóstico da situação atual do processo de desenvolvimento de software da empresa, foram utilizados os documentos para aplicação do QDPS-N2. O questionário foi respondido pelo diretor juntamente com os gerentes das áreas de desenvolvimento e de laboratório de WEB. Após a avaliação das respostas do questionário, foi elaborado o Relatório de Atividades de acordo com as diretrizes para o estabelecimento de melhoria, comentadas na Seção 3.3, contendo as práticas-chaves que empresa não realiza e que deveria realizar para conseguir satisfazer as KPA's do nível 2 do SW-CMM.

Com base nessas respostas constatou-se que a empresa já conhecia o SW-CMM e pretendia utilizá-lo para a melhoria de seu processo, visualizando os benefícios decorrentes dessa decisão, que certamente iriam culminar com o aumento da produtividade e lucro. Já havia uma pequena preocupação em medir a qualidade do processo de software, embora de maneira completamente informal e baseada na satisfação e reclamações dos clientes. Percebeu-se que a empresa atendia mais fortemente a KPA de Gerenciamento de Requisitos, um pouco das KPA's de Planejamento de Projeto de Software e de Acompanhamento e Supervisão de Projeto de Software e nada das KPA's Garantia de Qualidade de Software e Gerenciamento de Configuração de Software. A empresa não possui Subcontratados.

\section{- Definição do processo atual da empresa}

Essa atividade foi realizada em reuniões com o diretor e sua equipe. $O$ processo foi modelado utilizando-se Diagramas de Fluxo de Dados (DFD) [10], por ser essa a técnica de conhecimento comum de toda a equipe. Em relação à terminologia, ficou estabelecido que continuaria a ser usada a mesma que a empresa já utilizava e que possui os seguintes termos: processo é o termo usado para se referir ao processo como um todo; procedimento é o termo utilizado para se referir a cada processo do DFD e tarefa é o termo utilizado para se referir às tarefas (passos) de cada procedimento. Uma parte do processo que foi identificado inicialmente está representada na Figura 1.

Como pode ser visto na Figura 1, na parte inferior de cada procedimento está definido o responsável pelo procedimento, sendo que estes podem ser: a área comercial (Comercial), o grupo de desenvolvimento (Desenv) ou o grupo de desenvolvimento de layout (LabWeb).

Cada procedimento foi identificado e descrito de forma pormenorizada utilizando-se o formulário apresentado na Figura 2, iniciando-se assim a documentação do processo da empresa. O formulário contém campos de preenchimento para identificação do procedimento, seus objetivos, pessoas ou departamento responsável pelo procedimento, e as tarefas necessárias para a execução completa do mesmo.

Os critérios de inicialização e os elementos de entrada definem as pré-condições para que um procedimento possa ser executado.

Pensando na etapa de melhoria do processo, esse formulário foi preenchido visando o registro e/ou sugestão de critérios de finalização e utilização de métricas que eram ou poderiam ser usadas como pontos de verificação relativos às atividades de Gerenciamento de Qualidade de Software (GQS). 


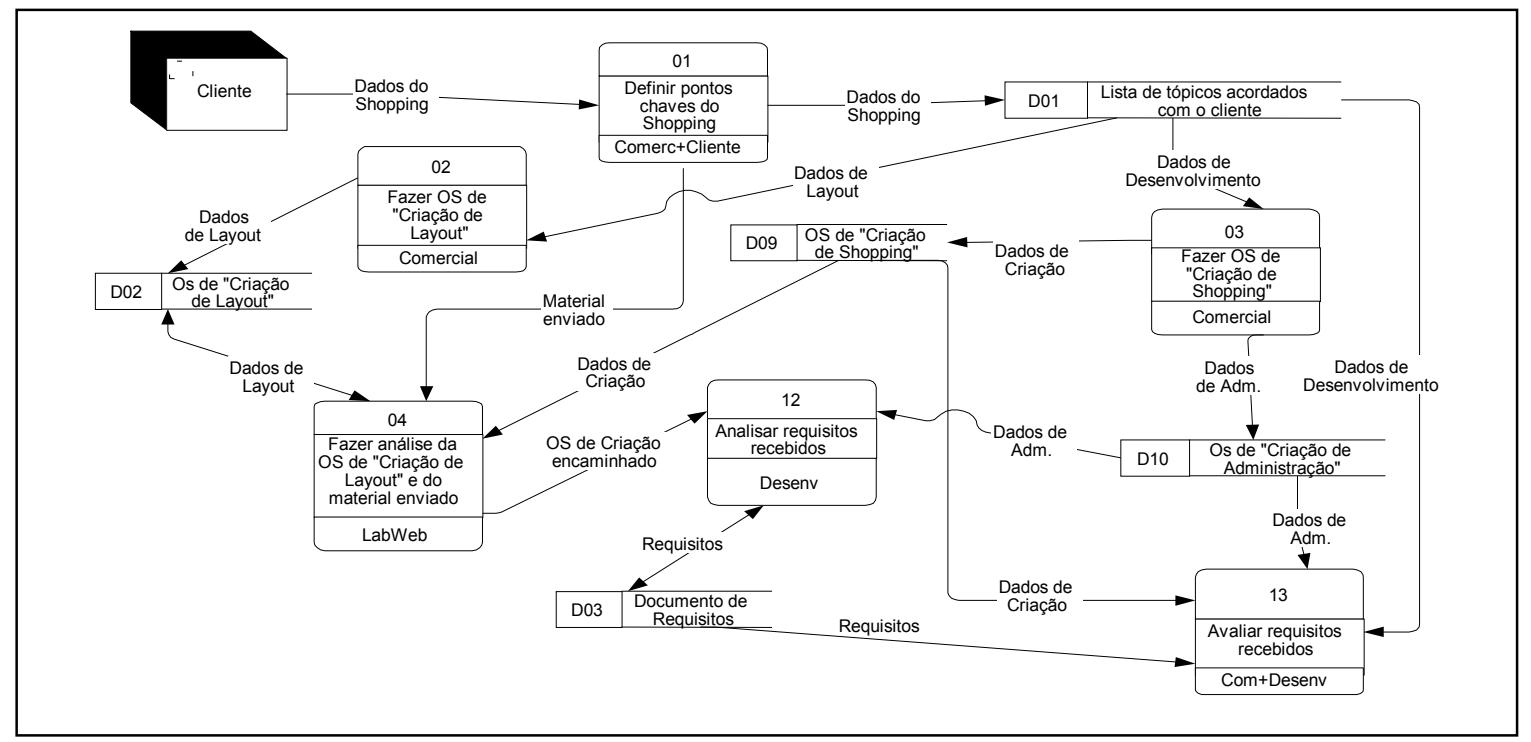

Figura 1. Parte do processo definido inicialmente

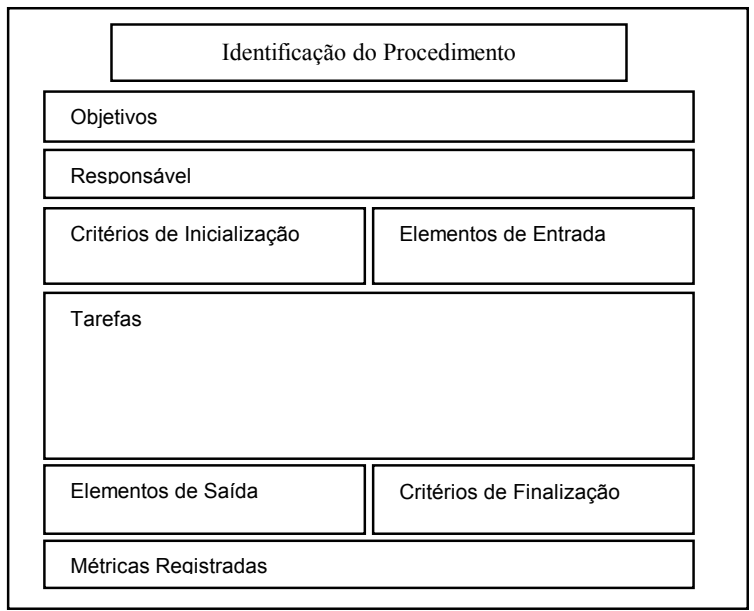

Figura 2. Formulário de documentação de procedimento

À medida que o DFD foi sendo construído e os formulários foram sendo preenchidos, alguns pontos isolados de melhoria foram facilmente observados; tais pontos estão comentados na próxima seção.

\subsection{Melhorias decorrentes da identificação do processo atual da empresa}

Ao se definir o processo atual da empresa perceberam-se alguns pontos, de caráter operacional, que poderiam ser melhorados alterando-se a forma de realização de tarefas específicas. Como essas tarefas apresentavam soluções alternativas que poderiam ser facilmente implementadas, decidiu-se realizar essas mudanças antes mesmo de se propor um plano de melhoria de processo mais amplo, gerado pelas diretrizes apresentadas no Item 3.3. Isso gerou um processo com algumas melhorias de ordem prática o qual está representado na Figura 3. 
Como exemplo das alterações, pode-se citar que foi percebido que a reunião de definição de requisitos (Procedimento 01 da Figura 1) não era muito proveitosa, pois o cliente não estava preparado e/ou munido de informações necessárias para que a empresa pudesse dar continuidade ao serviço. Assim, o processo seria mais eficiente e menos sujeito a iterações se o cliente já tivesse em mãos todos os documentos que ele achasse necessário para a definição do site solicitado (folders, imagens, logos, cores e outros). A solução foi simplesmente informar ao cliente durante o contato inicial, geralmente feito por telefone, que ele deveria estar portando tais documentos. Para representar essa alteração, criou-se do Procedimento OO (Figura 3), anterior à reunião de definição de requisitos, que informa ao cliente quais documentos ele deve ter em mãos no momento dessa reunião.

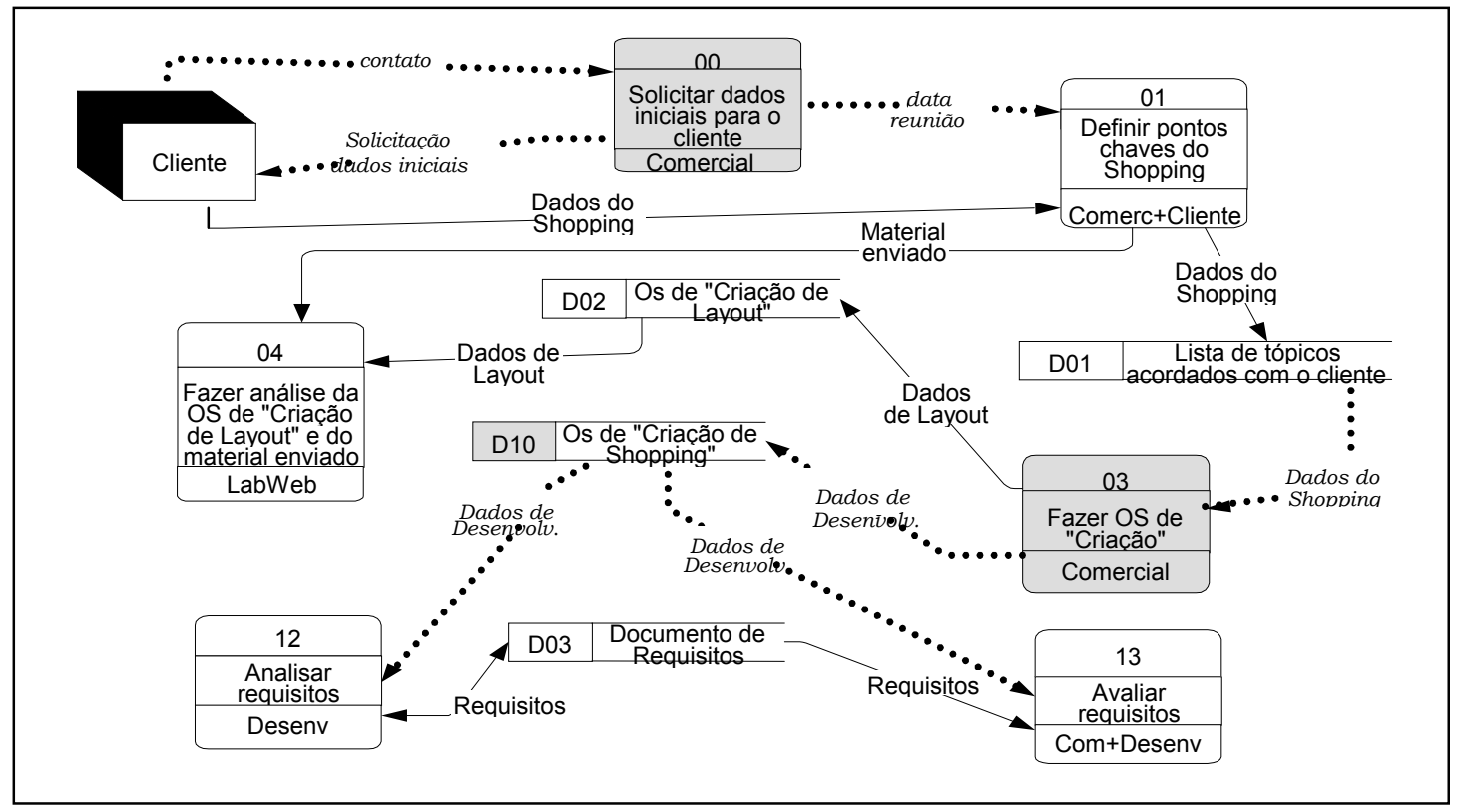

Figura 3. Pequenas melhorias no processo definido inicialmente

Um outro exemplo está relacionado ao envio de ordens de serviços. O departamento Comercial enviava três ordens de serviço, sendo que duas delas eram destinadas ao Desenvolvimento (na Figura 1, elaboradas pelo Procedimento 03 e colocadas uma no Depósito de Dados D09 e outra no D10). Ficou decidido que poderia ser gerada uma única ordem de serviço, contendo as informações das duas. Desse modo, os Depósitos de Dados D09 e D10 da Figura 1 se transformaram no Depósito de Dados D10 da Figura 3. Ainda em relação a essas ordens de serviço, havia o problema de que quem possuía o acesso ao Depósito de Dados D09, da Figura 1, era o LabWeb, o qual tinha que repassar a ordem de serviço para o Desenvolvimento pois, na realidade, essa ordem de serviço era para esse outro departamento. A solução foi modificar esse acesso, de forma que o Desenvolvimento pudesse acessá-la diretamente (fluxo de D10 para 12).

Um outro problema que foi verificado em relação ao envio das ordens de serviços, é que elas eram elaboradas por dois procedimentos diferentes (na Figura 1, o Procedimento 02 elabora a ordem de serviço para o LabWeb e o Procedimento 03 para o Desenvolvimento). Com isso, essas ordens de serviços eram, em geral, preparadas em momentos diferentes, ocasionando um atraso no prazo estipulado para a entrega do produto, ou, no mínimo, um trabalho sob muita pressão por parte de um dos dois departamentos, pois para definir o prazo de entrega era considerado que os departamentos iniciavam o trabalho simultaneamente. Para 
resolver esse problema, os envios das ordens de serviços passaram a ser tarefas de um único procedimento (Figura 3, Procedimento 03 - que é a união dos Procedimentos 02 e 03 da Figura 1) e, dessa forma, ambos os departamentos iniciam seus trabalhos ao mesmo tempo.

Além dessas alterações comentadas, também foram feitas algumas outras, em pontos isolados do processo definido inicialmente. No entanto, ressalta-se que essas pequenas melhorias foram adotadas com o objetivo específico de aumentar a eficiência do processo e não estavam direcionadas a atender nenhuma KPA em particular, uma vez que, em relação às diretrizes para o estabelecimento de melhoria, apenas a primeira etapa estava sendo concluída.

Durante as reuniões para a definição do processo, constatou-se que a LinkWay utilizava um software para acompanhamento da produção da equipe, desenvolvido por eles próprios e inspirado em algumas características do modelo PSP [11]. Esse software foi modificado para agregar as sugestões e idéias que surgiram durante a etapa de identificação do processo permitindo que a empresa tivesse uma ferramenta automatizada para acompanhar toda a produção de sua equipe.

Ao término desse passo, a empresa passou a ter um processo de desenvolvimento de software bem definido, com algumas atividades sendo feitas de forma mais eficiente em relação à sua situação inicial. Foi então sugerido que esse processo fosse seguido durante o desenvolvimento de um novo site a fim de verificar se estava realmente consistente.

Ao término desse passo, a empresa passou a ter um processo de desenvolvimento de software bem definido, com algumas atividades sendo feitas de forma mais eficiente em relação à sua situação inicial. Foi então sugerido que esse processo fosse seguido durante o desenvolvimento de um novo site a fim de verificar se estava realmente consistente.

\subsection{Utilização do Processo Definido}

Após a definição do processo, que a partir desse ponto será chamado de processo atual da empresa, o mesmo foi utilizado para desenvolver um site com o objetivo principal de fazer uma consistência do processo abstraído, além de verificar as vantagens/desvantagens das pequenas alterações sugeridas até então. Essa experiência foi muito positiva, principalmente em relação à data de entrega do produto. Notou-se que algumas das sugestões feitas à empresa, por ocasião do preenchimento do formulário de documentação dos procedimentos, facilitaram muito o levantamento dos requisitos, o que tem uma implicação direta na data de entrega do produto. Dessa forma, os departamentos de Desenvolvimento e LabWeb trabalharam sem muita pressão e com o cronograma melhor definido. Como o software de acompanhamento da equipe passou a retratar o processo atual, ficou mais fácil definir as tarefas atribuídas a cada funcionário bem como as responsabilidades de cada departamento, melhorando, dessa forma, o relacionamento e a integração dos mesmos. A existência da documentação de cada procedimento, disponível em rede pelo próprio software de acompanhamento, fez com que diminuísse o tempo de treinamento e as dúvidas dos funcionários, pois o aprendizado foi facilitado. Constatou-se também que o ânimo das equipes melhorou sensivelmente, pois o Departamento Comercial ficou satisfeito por entregar o produto na data combinada e os departamentos de Desenvolvimento e LabWeb conseguiram definir o cronograma e seguí-lo como planejado.

\subsection{Planejamento da melhoria}

A partir dos resultados obtidos até então com o diagnóstico e definição do processo 
atual, o passo seguinte foi aplicar as diretrizes para estabelecimento de melhoria, apresentadas na Seção 3.3. Como já foi mencionado, foi elaborado o Relatório de Atividades como resultado da etapa de diagnóstico.

A empresa também respondeu o Questionário de Pré-Condições e, após a análise dos dados, foi elaborado o Relatório de Pré-Condições contendo as pré-condições que a empresa não possui e que são necessárias para que ela consiga atender as KPA's do nível 2. Em seguida, o Relatório de Prioridades foi elaborado com base nas respostas positivas do Questionário de Pré-Condições e seu conteúdo refere-se às KPA's que podem ser mais facilmente atingidas, pois possuem maior número de pré-condições já atendidas pela empresa. Foi constatado que a empresa possui todas as pré-condições para a realização das KPA's de Planejamento de Projeto de Software e de Acompanhamento e Supervisão de Projeto de Software, $75 \%$ das pré-condições para a realização da KPA de Gerenciamento de Requisitos e nenhuma pré-condição para a realização das KPA's Garantia de Qualidade de Software e Gerenciamento de Configuração de Software.

Com base nessas informações e também em seus objetivos de negócios, a empresa definiu as prioridades das atividades de melhoria a serem executadas na seguinte ordem: atender em primeiro lugar a KPA de Gerenciamento de Requisitos; em segundo a KPA de Planejamento de Projeto de Software e, em terceiro, a KPA de Acompanhamento e Supervisão de Projeto de Software. Embora algumas iniciativas para a definição de atividades de Garantia de Qualidade de Software já tenham sido tomadas quando da definição e descrição dos procedimentos no formulário da Figura 2, essa KPA e a de Gerenciamento de Configuração de Software serão tratadas após as outras que foram estabelecidas como prioridade inicial da empresa.

Foi então gerado o Plano de Ação contendo as atividades do Relatório de Atividades e do Relatório de Pré-Condições, que discriminam as atividades a serem executadas pela empresa de acordo com as prioridades estabelecidas para as KPAs.

\section{Lições Aprendidas}

Durante a realização do Estudo de Caso, algumas dificuldades foram enfrentadas, principalmente na adaptação do ferramental para que este pudesse ser usado em conjunto. Mas, a principal lição tirada deste trabalho foi em relação à etapa de definição de processo pois, durante essa etapa, os pequenos problemas que foram sendo verificados e que poderiam ser resolvidos com pequenas mudanças na forma de realizar as atividades, já foram sendo corrigidos pela empresa. Pelo lado da empresa, essas pequenas melhorias funcionaram como incentivo, pois eles já podiam sentir seus resultados, de forma quase que imediata nas tarefas do dia a dia. No entanto, do ponto de definição do processo inicial de desenvolvimento, a implementação dessas melhorias dificultou essa atividade pois, embora pequenas, de certa forma descaracterizaram o processo relativo ao momento em que o trabalho começou. Assim, aparentemente, a atividade de definição do processo parece se tornar mais eficiente caso todas as melhorias que sejam observadas, sejam comentadas após o término dessa tarefa.

Outro ponto que se verificou foi que depois que o trabalho foi concluído, a empresa não está mantendo o mesmo ritmo com que respondia às solicitações decorrentes do trabalho de melhoria. Ou seja, para que a melhoria do processo realmente aconteça com a mesma predisposição inicial da empresa, é fundamental a figura de um "gerenciador da qualidade" que não deixe o ânimo reduzir. 


\section{Conclusões}

Em relação às expectativas da empresa, percebeu-se que a definição do processo inicial e as pequenas alterações realizadas nesse processo, gerando o processo atual de desenvolvimento de software, provocou melhorias que puderam ser sentidas de imediato pelos funcionários da empresa. Isso contribui bastante para a mudança cultural, facilitando os passos que virão em seguida, correspondentes às mudanças que serão necessárias para o atendimento das KPA's do nível 2 do CMM. Essas pequenas alterações que já foram incorporadas no processo, ocasionaram uma grande satisfação nos funcionários, os quais puderam trabalhar de forma mais organizada e com um cronograma melhor definido, o que influenciou diretamente no cumprimento do prazo de entrega do produto.

Quanto ao ferramental utilizado, percebeu-se que o uso em conjunto desses três trabalhos - o questionário QDPS-N2, a ferramenta SproQ e as Diretrizes para Iniciar a Melhoria de Processo - constitui uma estratégia que facilitou, na prática, os objetivos estabelecidos inicialmente de avaliar e propor um plano de melhoria para o processo de desenvolvimento de software da LinkWay.

\section{Referências Bibliográficas}

[1] Pfleeger,S.L.; Software Engineering - Theory and Practice, Prentice Hall PTR (1998).

[2] Falbo, R.A., Menezes, C.S., Rocha, A.R.C.; "Assist-Pro: Um Assistente Baseado em Conhecimento para Apoiar a Definição de Processos de Software”, anais do XIII Simpósio Brasileiro de Engenharia de Software, Florianópolis, (1999), pp. 147-162

[3] Machado, L.F.D.C., Santos, G., Oliveira, K.M., Rocha, A.R.C.; "Def-Pro: Apoio Automatizado para a Definição de Processos de Software", anais do XIV Simpósio Brasileiro de Engenharia de Software, João Pessoa, (2000), pp. 359 - 362

[4] Paulk, M.C., Curtis, B., Chrissis, M.B., Weber, C.V.; Capability Maturity Model for Software, version 1.1, Relatório Técnico, Software Engineering Institute, Carnegie Mellon University, Pittsburgh, Pennsylvania, (1993).

[5] ISO/IEC TR 15504, 1998, Parts 1-9: Information Technology-Software Process Assessment.

[6] Ministério de Ciência e Tecnologia - Qualidade e Produtividade no Setor de Software Brasileiro - Principais Resultados Históricos, Resultados da Pesquisa 1999. Acessado em 20/05/2002. Disponível na Internet: http://www.mct.gov.br/sepin

[7] Marchetti, P.A.S.; Uma Abordagem para Diagnóstico de Processo de Software Baseada no Nível 2 do CMM, Dissertação de Mestrado do Centro de Ciências Exatas e de Tecnologia, Universidade Federal de São Carlos, São Carlos, (2000)

[8] Gaspar, D.A.; Desenvolvimento de Uma Ferramenta de Apoio á Avaliação de Qualidade de Processo de Software para Pequena Empresa, Dissertação de Mestrado do Instituto de Ciências Matemáticas e de Computação, Universidade de São Paulo, São Carlos, (2000) [9] Fiats, M.I.; Diretrizes para o Estabelecimento de Melhoria de Processos Adequada a Empresas de Pequeno Porte, Dissertação de Mestrado do Instituto de Ciências Matemáticas e de Computação, Universidade de São Paulo, São Carlos, (2000)

[10] Gane, C., Sarson, T.; Structured System Analysis, Prentice Hall, (1979)

[11] Personal Software Process. Acessado em 20/05/2002. Disponível na Internet: http://www.sei.cmu.edu/tsp/psp.html 